\title{
La teoría de los sistemas complejos como aporte alternativo para comprender el proceso de formación de la isla de calor en la ciudad de Presidente Prudente (San Pablo, Brasil)
}

The theory of complex systems as an alternative contribution to understanding the process of formation of the heat island in the city of Prudent President (Sao Paulo, Brazil)

\author{
María Cecilia Domizio \\ Universidad Nacional de Cuyo (UNCUYO) \\ Argentina \\ D.Jeciliadomizio@ffyl.uncu.edu.ar
}

\section{RESUMEN}

En la ciudad de Presidente Prudente, situada en el oeste del estado de San Pablo (Brasil), se han realizado en las últimas décadas estudios pioneros para constatar la existencia de la isla de calor en una urbe de tamaño poblacional medio, lo que tradicionalmente se investigaba en aglomerados urbanos. Estos trabajos, que abordan la isla de calor urbana como un hecho mensurable, pueden complementarse con un aporte alternativo para profundizar en su conocimiento. Por eso, el presente trabajo observa este fenómeno climático desde la Teoría de los Sistemas Complejos, que permite analizar su constitución desde sus principales componentes y sus relaciones espacio-temporales, lo cual permite conocer la formación y funcionamiento de la isla de calor prudentina como sistema. Esta visión corrobora la presencia del fenómeno, pero en su evolución surgen desfasajes en algunos aspectos, que lleva a formular interrogantes y recurrir a los estudios realizados para abrir nuevos caminos de investigación.

PALABRAS CLAVE: isla de calor urbana, teoría de los sistemas complejos, ciudades intermedias, evolución de fenómeno urbano 


\begin{abstract}
In the city of Presidente Prudente, located in the western state of São Paulo (Brazil), pioneering studies have been carried out in recent decades to verify the existence of the heat island in a city of medium population size, what was traditionally researched in urban agglomerates. These works, which consider urban heat island as a measurable fact, can be complemented with an alternative contribution to deepen their knowledge. Therefore, the present work observes this climatic phenomenon from the Theory of Complex Systems, which allows analyzing its constitution from the main components and its spatial-temporal relations, which allows to know the formation and operation of the prudentine heat island as a system. This vision corroborates the presence of the phenomenon, but in this evolution, there are gaps in some aspects, which leads to the formulation of questions and the use of studies carried out to open new ways of research.
\end{abstract}

KEYWORDS: urban heat island, theory of complex systems, intermediate cities, evolution of urban phenomenon.

\title{
INTRODUCCIÓN
}

Presidente Prudente es una ciudad de porte medio, que hacia el año 2010 tenía una población de 203.375 habitantes. Aunque su tamaño poblacional no es muy significativo, la urbe prudentina sufrió a mediados de siglo XX una importante expansión de su trama, que llevó a la transformación radical de su sitio. De este modo, la sustitución de la vegetación primitiva por construcciones, la impermeabilización del suelo y el desarrollo de actividades humanas, sobre todo la industria, el transporte intraurbano y el consumo energético edilicio, han causado un aumento de las temperaturas en la ciudad.

Este conjunto de hechos llevó a la formación de la isla de calor en Presidente Prudente, lo cual fue constatado a través de numerosas investigaciones (Amorim, M., 2000; Menotti et al., 2004; Amorim, M., Sant'Anna Neto, J. \& Dubreuil, V., 2008; Cardoso, R., 2015). Se trata de estudios innovadores y relativamente recientes, ya que este fenómeno climático urbano hasta el momento solo se había investigado en grandes ciudades de Brasil. En general, en estos trabajos científicos predomina una visión analítica y el uso de técnicas cuantitativas para comprobar la existencia concreta del objeto estudiado. 
Este abordaje es válido y necesario, especialmente por el carácter tangible del fenómeno urbano. Asimismo, es posible complementar este enfoque con otro alternativo para profundizar y enriquecer las investigaciones. Entre ellos, se encuentra el aporte de la Teoría de los Sistemas Complejos, cuya diferencia con los estudios tradicionales radica en analizar un fenómeno estableciendo múltiples relaciones entre aspectos en distintos niveles espaciales y temporales. Este análisis permite lograr una visión más amplia y una explicación más completa de la realidad en cuestión.

Por eso, el presente trabajo aborda desde el enfoque sistémico complejo la formación de la isla de calor en la ciudad de Presidente Prudente, considerando la influencia de aspectos significativos, como el crecimiento demográfico, la expansión de la malla urbana, el retroceso del medio natural y la presencia de arbolado urbano. A partir de este conocimiento será posible ampliar la comprensión de este fenómeno urbano, visualizar escenarios futuros y formular preguntas reveladoras para abrir nuevas líneas de investigación.

\section{Marco teórico-metodológico para el estudio de la isla de calor urbana como fenómeno complejo}

El análisis de la isla de calor urbana desde el enfoque sistémico complejo requiere partir del concepto de sistema: una totalidad que emerge de las interacciones establecidas entre sus componentes, de modo que supera la suma de sus elementos (Codes, I., Robledo, S., 1997). Además, el sistema es un ente dinámico, que supone un continuo intercambio de materia y energía con el entorno, lo cual le permite a este evolucionar, es decir, pasar de un estado a otro.

Así, la isla de calor urbana puede considerarse como un sistema que surge de las relaciones entre elementos diferentes, que se extraen de su definición conceptual: bolsón de aire caliente formado en los ambientes urbanos, que genera una diferencia de temperatura con el entorno rural cercano, debido a la modificación antrópica del medio natural (Amorim, M., 2017). También incluye las diferencias térmicas intraurbanas, originadas de los distintos usos y coberturas del suelo, de la vegetación, del relieve, de la geometría y densidad de las edificaciones, de los 
materiales constructivos y del flujo de calor generado por las actividades humanas (Amorim, M., 2010, Cardoso, R., 2015).

Entre estos componentes, la población, la trama urbana, la construcción edilicia, la arboleda de calle, el medio rural inmediato y la temperatura de la ciudad resultan importantes para la construcción del sistema isla de calor urbana de Presidente Prudente (en adelante sistema ICUPP). Pero la conformación de este sistema excluye la práctica habitual de unir todas las variables y entrelazarlas (García, R., 2006). Por eso, es necesario profundizar en el enfoque sistémico tradicional con las contribuciones teórico-metodológicas de la Teoría de los Sistemas Complejos.

Esta teoría avanza un poco más al considerar las múltiples facetas y dimensiones del sistema, así como la interdependencia de todos sus componentes, lo cual da el carácter de complejo al sistema. Así, se analizan simultáneamente varios tipos de relaciones: entre elementos del sistema, entre el sistema y su entorno y entre distintas escalas espaciales y temporales por las que transcurre el sistema (Domizio, M. C., 2018). Pese a que esta concepción puede aparentar un estudio intrincado, más bien implica una acotada serie de pasos a fin de simplificar y comprender el complejo empírico analizado.

El primer paso consiste en separar el sistema elegido del resto de la realidad mediante la definición de sus aspectos constitutivos y sus relaciones, lo cual en el caso del sistema ICUPP ya fue mencionado. El segundo paso comprende elegir las escalas espacio- temporales en las que ocurre el sistema para conocer los procesos más importantes que llevaron a su estado actual. En este estudio, los elementos del sistema ICUPP suceden en un nivel espacial, la ciudad de Presidente Prudente, y en diferentes niveles temporales, que corresponden a los períodos históricos de la urbe desde 1917 a 2016.

El tercer y último paso supone deducir del análisis de los procesos la estructura del sistema, es decir, el entramado permanente de relaciones entre los elementos de la totalidad, ya que esa estructura se va formando a medida que ocurren las interacciones en el tiempo. Esta instancia es la parte más importante del presente estudio, porque incluye la descripción histórica de la realidad observada, el análisis de los procesos concernientes al sistema ICUPP y la construcción de su estructura. Una vez alcanzado el sistema, es posible observar el grado de participación de cada 
parte e identificar aquellas que necesiten estudios especializados a fin de profundizar en la problemática.

\section{Sitio de Presidente Prudente}

Presidente Prudente es un municipio brasileño del interior del Estado de San Pablo, que dista $558 \mathrm{~km}$ de la capital estadual homónima y ocupa una superficie de 562,97 $\mathrm{km}^{2}$. El municipio está formado por cinco distritos: Ameliópolis, Eneida, Floresta do Sul, Montalvão y distrito sede, donde se encuentra la ciudad de Presidente Prudente, que constituye el principal centro urbano del municipio y de la región oeste de San Pablo, Figura 1.

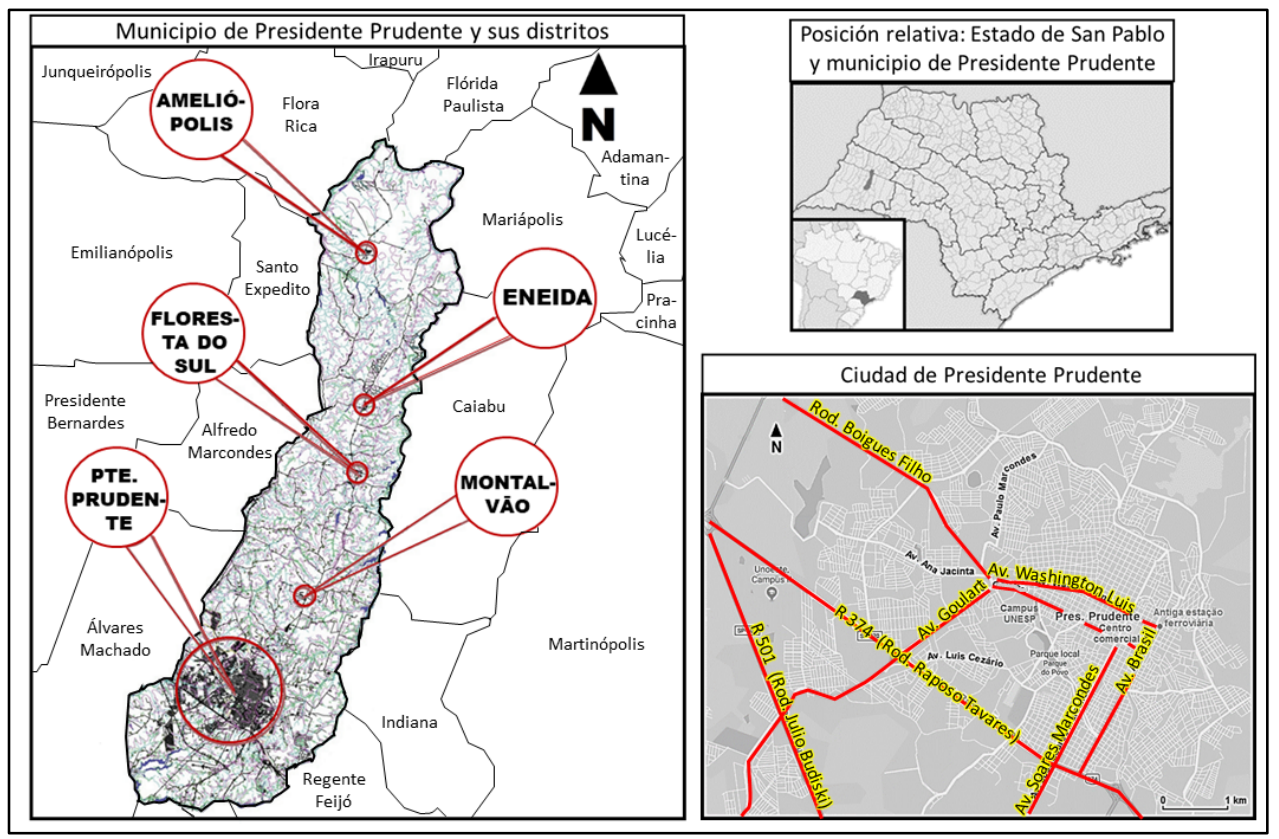

Figura 1. Municipio de Presidente Prudente, sus distritos y la ciudad homónima Fuente: Elaboración propia, sobre la base de Secretaría Municipal de Planeamiento, Desarrollo Urbano y Habitación, Prefectura de Presidente Prudente (2005), Wikipedia y Google Maps (2017). 
Desde el punto de vista geomorfológico, el territorio prudentino corresponde a la unidad morfoestructural llamada Planalto Occidental Paulista, que comprende formas de relieve surgidas de procesos erosivos, como colinas mayores de cumbres tabulares y colinas menores de cumbres convexas (Nunes, J., Freire, R., Peres, I., 2006). El área urbana está ocupada en gran parte por estas colinas, las cuales tienen entre 300 y $500 \mathrm{~m}$ de altura y una pendiente que varía entre 2 y $20 \%$. Entre sus vertientes existen cursos de agua, que pertenecen a la cuenca del río Paraná y en la ciudad se hallan canalizados.

En el centro-oeste paulista domina un clima tropical continental subhúmedo con rasgos de transición, producto de la entrada de diferentes masas de aire. Así, se observa un período cálido y lluvioso (de octubre a marzo), donde suceden los sistemas atmosféricos tropicales (masa tropical Atlántica, masa tropical Continental y masa Ecuatorial Continental) y se caracteriza por una temperatura media mensual de $24^{\circ} \mathrm{C}$ y la ocurrencia del $70 \%$ del total anual de precipitaciones $(1300 \mathrm{~mm})$. En el período ameno y seco (de abril a septiembre) acontecen los sistemas extratropicales (masa polar Atlántica) y comprende una temperatura media de $20^{\circ} \mathrm{C}$ y el $30 \%$ de las lluvias (Amorim et al., 2010).

Por último, el bioma de la región pertenece a la Mata Atlántica, que está formada por una vegetación aluvial y submontana, propia de la influencia de un clima estacional, que determina la caída parcial del follaje (Veloso, H., Rangel Filho, A., Lima, J., 1991). En la actualidad, esta floresta primitiva solo ocupa el $12,98 \%$ del territorio prudentino y se halla distribuida en áreas reducidas, dispersas y $\sin$ conexión entre sí, lo cual aumenta la fragilidad de estos ecosistemas naturales frente a acciones humanas como incendios, sobrepastoreo, etc.

\section{Evolución de la ciudad de Presidente Prudente en sus aspectos socioambientales}

La descripción histórica de Presidente Prudente en sus principales aspectos sociales y ambientales constituye el primer paso del análisis sistémico complejo para comprender y explicar la formación de la isla de calor urbana. A continuación, se considera cada etapa del desarrollo urbano prudentino, desde la fundación de la ciudad en 1917 hasta 2016, año anterior a la realización del presente estudio. 


\section{7-1959: Fundación de la ciudad y sus primeros desarrollos}

El origen de Presidente Prudente se vincula al avance de las áreas de plantación de café hacia el Ilamado "sertão" (interior) del estado de San Pablo, en un movimiento de colonización de este a oeste, incrementado por la necesidad de ocupar nuevas tierras, la presencia de mano de obra inmigrante y la suba de los precios del café en el mercado internacional. A este proceso ayudó fuertemente el tendido de las líneas del ferrocarril Sorocabana al permitir la circulación de personas y mercaderías (Whitacker, A., 2017).

Según Abreu (1972, citado por Whitacker, 2017), Prudente surgió de dos núcleos urbanos cuya función era sustentar la colonización agrícola (abastecimiento de instrumental de trabajo, alimentos, etc.), pero se fueron diferenciando en el tiempo. Así, la villa Goulart, fundada en 1917 hacia el oeste del ferrocarril, fue asumiendo las características propias del centro de la ciudad, debido a que la topografía suave de su sitio facilitaba el desarrollo urbano. Por su parte, la villa Marcondes, fundada en 1919 hacia el este de la línea férrea, pasó a ser lugar de soporte para la ocupación de tierras rurales, ya que el dominio de colinas con fuerte pendiente (de 10 a más de $20 \%$ ) dificultaba la urbanización, Figura 2.

Esta diferencia entre centro y periferia no solo fue funcional, sino que también se tornó social, ya que las acciones municipales de promoción urbana se dirigieron inicialmente al primer sector. El crecimiento de la ciudad fue escaso hasta 1940, cuando la trama urbana, orientada por la ferrovía, comenzó a extenderse en sentido norte-sur hasta ocupar los fondos de valle, donde existían arroyos, como do Bacarim, do Veado, etc. A mitad de 1950 aumentaron los loteos implantados en forma discontinua, lo que llevó a conformar grandes vacíos urbanos, al tiempo que se acentuaba la diferenciación socio-espacial.

Por otro lado, entre 1945 y 1962 el área de vegetación primitiva experimentó un retroceso importante, al pasar del $96 \%$ al $60 \%$ de la superficie total del municipio. Esto se debió a la expansión de las zonas de pastaje para el ganado bovino, el cual sustituyó a los cultivos de algodón y café y adquirió un papel fundamental en la economía prudentina (Leite, 1981, citado por Amorim et al., 2010). Además, según el Instituto Brasilero de Geografía y Estadística, a principios de 1960 el municipio 
presentaba una población de 72.782 habitantes, la mayor parte de la cual habitaba en el campo.

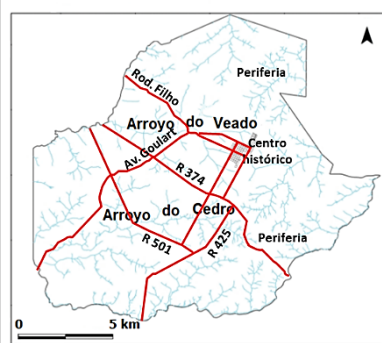

Malla urbana $1917-1923$

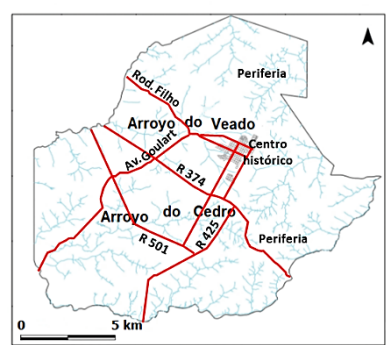

Malla urbana $1924-1940$

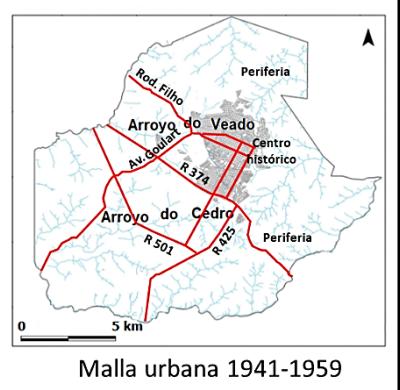

Figura 2. Expansión de la malla urbana de Presidente Prudente desde 1917 hasta 1959. Fuente: Elaboración propia, sobre la base de Whitacker, A. (2017).

\section{0-1999: Fuerte expansión urbana}

A partir de 1960 la ciudad de Prudente sufrió un significativo aumento poblacional, debido a la migración rural-urbana, por la que arribaron numerosos trabajadores de campo que quedaron desocupados por la práctica de la ganadería extensiva, y la migración urbana-urbana, por la cual llegaron habitantes de otras regiones paulistas. Así, la población urbana del municipio pasó de 92.601 a 129.624 habitantes entre 1970 y 1980, lo que representa un aumento del $40 \%$ en este período y afectó especialmente a la capital municipal, llevando a una importante expansión territorial (Pedro, L., 2014).

En este contexto, los fondos de valle y las abruptas colinas de los sectores este y norte fueron ocupados por la población recién llegada de bajo poder adquisitivo, Figura 3. Entre 1970 y 1980 continuó esta situación de segregación socio-espacial, porque el gobierno, mientras construía barrios populares en ambientes deteriorados (arroyos contaminados, zonas de inundación, etc.) del oeste, seguía otorgando los beneficios de la urbanización al sector ya consolidado (villa Goulart) y algunas partes de la periferia. En estos últimos espacios se desarrolló la actividad comercial e industrial (Sposito, 1983, citado por Pedro, 2014). Al mismo tiempo, debido al avance 
agrario, el área natural llegó al 5\% de la superficie total del municipio (Caccia Gouveia, J., Paes, J., 2017).

La transformación del medio natural circundante, producto de la expansión urbana y rural, fue conllevando un cambio en las temperaturas máximas y mínimas en la ciudad de Presidente Prudente. Según la estación meteorológica de la Universidad Estadual Paulista, entre 1970 y 1980 la media de las temperaturas máximas pasó de $28,79^{\circ} \mathrm{C}$ a $28,92^{\circ} \mathrm{C}$ y la media de las temperaturas mínimas pasó de $17,56^{\circ} \mathrm{C}$ a $18,41^{\circ} \mathrm{C}$.

En el estudio de Fante (2014) se mide con mayor precisión este cambio de temperaturas ocurrido en la urbe prudentina entre 1960 y 2011 a través del método de regresión lineal. Así, en el período 1961-1969 las temperaturas máximas aumentaron $0,08^{\circ} \mathrm{C}$, al pasar de $28,88^{\circ} \mathrm{C}$ a $28,96^{\circ} \mathrm{C}$, mientras las temperaturas mínimas se incrementaron en $0,52^{\circ} \mathrm{C}$, al pasar de $16,48^{\circ} \mathrm{C}$ a $17^{\circ} \mathrm{C}$. En el período siguiente, $1969-1979$, las temperaturas máximas aumentaron $0,12^{\circ} \mathrm{C}$, al pasar de $28,96^{\circ} \mathrm{C}$ a $29,08^{\circ} \mathrm{C}$, y las temperaturas mínimas ascendieron a $0,62^{\circ} \mathrm{C}$, al pasar de $17^{\circ} \mathrm{C}$ a $17,62^{\circ} \mathrm{C}$.

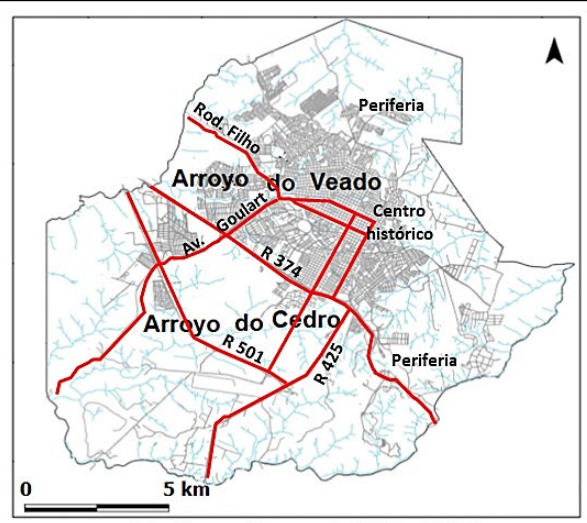

Malla urbana 1960-1982

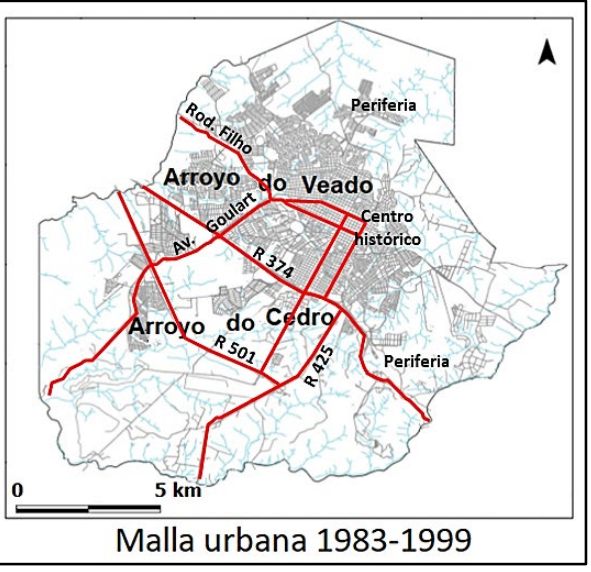

Malla urbana 1983-1999

Figura 3. Expansión de la malla urbana de Presidente Prudente desde 1960 hasta 1999 Fuente: Elaboración propia, sobre la base de Whitacker, A. (2017).

En la década de 1990 al crecimiento urbano se agregó la construcción vertical, ubicada especialmente en el sector céntrico. Además, continuó la extensión de las 
periferias mediante la creación de espacios de segregación y autosegregación. Los primeros han comprendido áreas de baja renta, con alto riesgo natural (infiltraciones de agua e inundaciones), distantes y de difícil acceso a servicios públicos y equipamientos. Los segundos han constituido espacios residenciales cerrados de alta renta, un poco alejados del centro urbano, pero conectados a través de carreteras (Whitacker, A., 2017).

Por su parte, la población urbana solo creció un $24 \%$ entre 1980 y 1990, tendencia decreciente que se mantuvo en el próximo período, 1990-2000, cuando dicho aumento poblacional fue solo de $16 \%$. Mientras, continuaron los cambios de temperatura en la ciudad:

- entre 1980 y 1990 la media de las temperaturas máximas pasó de $28,92^{\circ} \mathrm{C}$ a $29,13^{\circ} \mathrm{C}$ y la media de las temperaturas mínimas pasó de $18,41^{\circ} \mathrm{C}$ a $18,74^{\circ} \mathrm{C}$. Según Fante (2014), entre 1979 y 1989 las temperaturas máximas aumentaron $0,12^{\circ} \mathrm{C}$, al pasar de $29,08^{\circ} \mathrm{C}$ a $29,2^{\circ} \mathrm{C}$, y las mínimas ascendieron $0,63^{\circ} \mathrm{C}$, al pasar de $17,62^{\circ} \mathrm{C}$ a $18,25^{\circ} \mathrm{C}$.

- entre 1990 y 2000 la media de las temperaturas máximas pasó de $29,13^{\circ} \mathrm{C}$ a $29,61^{\circ} \mathrm{C}$ y la media de las temperaturas mínimas pasó de $18,74^{\circ} \mathrm{C}$ a $19,1^{\circ} \mathrm{C}$. Según Fante (2014), entre 1989 y 1999 las temperaturas máximas aumentaron $0,08^{\circ} \mathrm{C}$, al pasar de $29,2^{\circ} \mathrm{C}$ a $29,28^{\circ} \mathrm{C}$, y las mínimas ascendieron $0,7^{\circ} \mathrm{C}$, al pasar de $18,25^{\circ} \mathrm{C}$ a $18,95^{\circ} \mathrm{C}$.

\section{0-2016: Crecimiento urbano desacelerado, pero complejo}

Después del año 2000 se desarrollaron varios loteos, sobre todo en intersticios urbanos (ej. fondos de valle) para construir aglomerados habitacionales destinados a población de baja condición socio-económica, lo cual contribuyó a la expansión de la trama urbana prudentina, pero sin la gravitación de décadas anteriores, Figura 5. A la par de este reducido crecimiento espacial, la ciudad ha desarrollado recientemente una complejidad poco tangible. Se trata de la ubicación de locales comerciales y de servicios (ej. shoppings, vías especializadas en ciertos rubros, etc.) en áreas periféricas, que han generado nuevas centralidades, atrayendo flujos de distinto tipo (Pereira, S., 2006). 
En cuanto a la población urbana, esta continuó con la tendencia decreciente del período anterior, al registrar un aumento de solo $10 \%$ entre 2000 y 2010 . Con respecto a los registros térmicos de la ciudad, se destaca, como único dato obtenido, que entre 2000 y 2010 las temperaturas máximas aumentaron $0,12^{\circ} \mathrm{C}$, al pasar de $29,28^{\circ} \mathrm{C}$ a $29,4^{\circ} \mathrm{C}$, y las mínimas ascendieron $0,65^{\circ} \mathrm{C}$, al pasar de $18,95^{\circ} \mathrm{C}$ a $19,6^{\circ} \mathrm{C}$.

Por otro lado, la arborización urbana contaba en 1995 con 147 espacios verdes, que comprendían una superficie de 1,86 km² (Gomes, M., Amorim, M., 2002), mientras en 2016 existían 116 áreas verdes, alcanzando una extensión de 1,76 km² (Souza, M., 2016). A nivel espacial, las zonas sur y oeste de Prudente han contado con mayor porcentaje de superficie verde y el dominio de árboles, en cambio las zonas norte y este han dispuesto de menor área vegetada, formada por hierbas o arboleda dispersa, Figura 4. A nivel social, los barrios que históricamente fueron valorizados por el sector público y privado han contado con una trama verde mejor desarrollada que los loteos de baja renta.

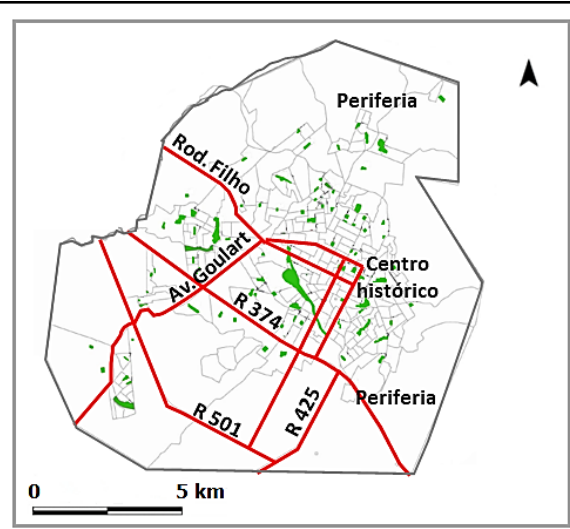

Áreas verdes urbanas (2016)

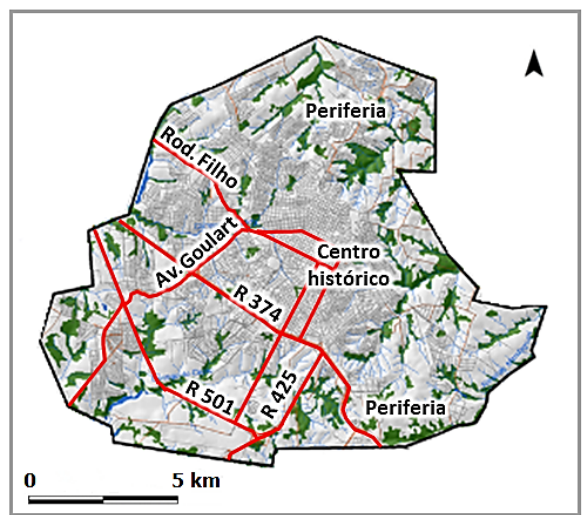

Áreas verdes naturales (2013)

Figura 4. Distribución de áreas verdes urbanas y naturales en Presidente Prudente Fuente: Elaboración propia, sobre la base de Souza, M. (2016) y Caccia Gouveia, J., Paes, J. (2017).

Finalmente, en la actualidad la densidad edilicia en el centro urbano y el área consolidada -construida hasta 1960- es alta, incluyendo muchas construcciones verticales, mientras la densidad demográfica es media o baja. Esto último indica el 
dominio en estos espacios de la función comercial y de servicios, que lleva a que su principal concurrencia sea en la semana (Cardoso, R., 2015). A la inversa, la periferia y urbanizaciones hechas después de 1960 tienen baja concentración de edificios y alta densidad poblacional, Figura 5. Allí domina la función residencial, que supone una gran afluencia durante el fin de semana.

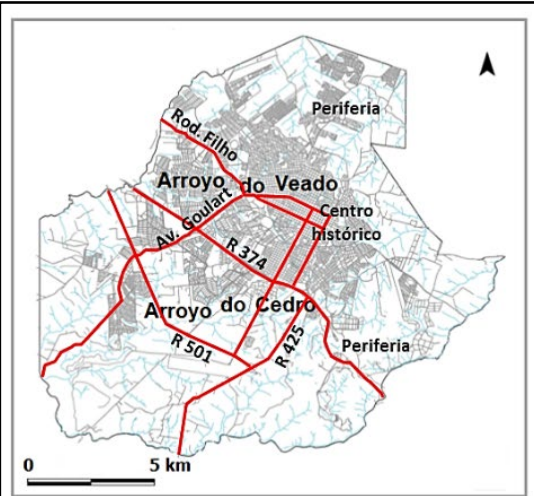

Malla urbana 2000-2016

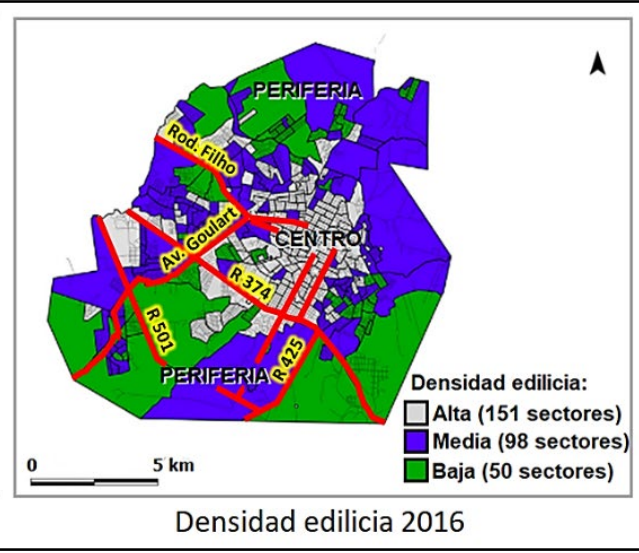

Densidad edilicia 2016

Figura 5. Expansión de la malla urbana entre 2000 y 2016 en Presidente Prudente y su densidad edilicia en 2016.

Fuente: Elaboración propia, sobre la base de Whitacker, A. (2017) y Melazo, E., Cardoso, J., Magaldi, S. (2017).

\section{Construcción del sistema isla de calor de Presidente Prudente}

Una vez conocida la evolución de la ciudad de Presidente Prudente en sus aspectos socio-ambientales - principales factores explicativos de la isla de calor urbana-, es posible extraer los procesos más importantes que llevan a la conformación del sistema ICUPP. Para ello, la siguiente tabla muestra el desarrollo de cada elemento del sistema en cada período histórico de la urbe prudentina. Las interrelaciones entre estos componentes permiten construir la estructura del sistema ICUPP desde su estado inicial hasta el actual y, de allí, observar la incidencia de cada parte en el funcionamiento del todo. 


\begin{tabular}{|c|c|c|c|c|c|}
\hline \multirow{2}{*}{$\begin{array}{c}\text { Elementos } \\
\text { del sistema } \\
\text { ICUPP }\end{array}$} & \multicolumn{5}{|c|}{ Períodos del desarrollo histórico de Presidente Prudente } \\
\hline & $1917-1940$ & 1941-1959 & $1960-1979$ & $1980-1999$ & 2000-2016 \\
\hline $\begin{array}{c}\text { Malla } \\
\text { urbana }\end{array}$ & $\begin{array}{l}\text { - Escaso creci- } \\
\text { miento } \\
\text { - Creación de } \\
\text { dos núcleos ur- } \\
\text { banos entorno } \\
\text { al ferrocarril So- } \\
\text { rocabana }\end{array}$ & $\begin{array}{l}\text { - Moderado cre- } \\
\text { cimiento } \\
\text { - Extensión ur- } \\
\text { bana alrededor } \\
\text { de los núcleos } \\
\text { iniciales, ocu- } \\
\text { pando colinas } \\
\text { bajas y forman- } \\
\text { do intersticios }\end{array}$ & $\begin{array}{l}\text {-Gran crecimi- } \\
\text { ento } \\
\text { - Consolidación } \\
\text { del centro urba- } \\
\text { no y alrededo- } \\
\text { res y extensión } \\
\text { urbana al norte, } \\
\text { este y oeste, } \\
\text { ocupando fon- } \\
\text { dos de valle e } \\
\text { intersticios }\end{array}$ & $\begin{array}{l}\text { - Escaso creci- } \\
\text { miento } \\
\text { - Extensión ur- } \\
\text { bana, ocupando } \\
\text { intersticios, ha- } \\
\text { cia el norte y } \\
\text { oeste (clase } \\
\text { baja) y hacia el } \\
\text { sur y oeste } \\
\text { (clase alta) }\end{array}$ & $\begin{array}{l}\text { - Escaso creci- } \\
\text { miento } \\
\text { - Creación de } \\
\text { nuevas centrali- } \\
\text { dades en perife- } \\
\text { ria mediante la } \\
\text { instalación de } \\
\text { locales comer- } \\
\text { ciales y de ser- } \\
\text { vicios }\end{array}$ \\
\hline $\begin{array}{c}\text { Población } \\
\text { urbana }\end{array}$ & $\begin{array}{l}\text { - Escasa } \\
\text { - Mayor parte } \\
\text { de la población } \\
\text { del municipio } \\
\text { habita en zona } \\
\text { rural }\end{array}$ & $\begin{array}{l}\text { - Escasa } \\
\text { - Mayor parte } \\
\text { de la población } \\
\text { del municipio } \\
\text { habita en zona } \\
\text { rural }\end{array}$ & $\begin{array}{l}\text { - Gran aumento: } \\
40 \% \text { entre } 1970 \\
\text { y } 1980 \\
\text { - Mayor parte } \\
\text { de la población } \\
\text { del municipio } \\
\text { habita en ciu- } \\
\text { dad capital }\end{array}$ & $\begin{array}{l}\text { - Aumento de- } \\
\text { creciente: } 24 \% \\
\text { entre } 1980 \mathrm{y} \\
1990 \text { y } 16 \% \\
\text { entre } 1990 \mathrm{y} \\
2000 \\
\text { - Mayor parte } \\
\text { de la población } \\
\text { del municipio } \\
\text { habita en ciu- } \\
\text { dad capital }\end{array}$ & $\begin{array}{l}\text { - Aumento de- } \\
\text { creciente: } 10 \% \\
\text { entre } 2000 \mathrm{y} \\
2010 \\
\text { - Mayor parte } \\
\text { de la población } \\
\text { del municipio } \\
\text { habita en ciu- } \\
\text { dad capital }\end{array}$ \\
\hline $\begin{array}{l}\text { Entorno } \\
\text { rural }\end{array}$ & $\begin{array}{l}\text { - Dominio de la } \\
\text { vegetación na- } \\
\text { tural }\end{array}$ & $\begin{array}{l}\text { - Gran defores- } \\
\text { tación para el } \\
\text { desarrollo agra- } \\
\text { rio (entre } 1945 \\
\text { y } 1962 \text {, el área } \\
\text { de vegetación } \\
\text { natural pasó de } \\
96 \% \text { a } 60 \% \text { del } \\
\text { área municipal) }\end{array}$ & $\begin{array}{l}\text { - Gran defores- } \\
\text { tación para el } \\
\text { desarrollo agra- } \\
\text { rio (el área de } \\
\text { vegetación na- } \\
\text { tural pasó a } 5 \% \\
\text { del área muni- } \\
\text { cipal) }\end{array}$ & $\begin{array}{l}\text { - Escasa defo- } \\
\text { restación por } \\
\text { consolidación } \\
\text { de la actividad } \\
\text { agraria }\end{array}$ & $\begin{array}{l}\text { - Escasa defo- } \\
\text { restación por } \\
\text { consolidación } \\
\text { de la actividad } \\
\text { agraria } \\
\text { - Áreas peque- } \\
\text { ñas con vege- } \\
\text { tación natural } \\
\text { arbustiva y ar- } \\
\text { bórea }\end{array}$ \\
\hline $\begin{array}{c}\text { Trama verde } \\
\text { urbana }\end{array}$ & $\begin{array}{l}\text { - Sin registro de } \\
\text { presencia de } \\
\text { áreas verdes }\end{array}$ & $\begin{array}{l}\text { - El desarrollo } \\
\text { de áreas verdes } \\
\text { es supuesto con } \\
\text { el desarrollo ur- } \\
\text { bano }\end{array}$ & $\begin{array}{l}\text { - El desarrollo } \\
\text { de áreas verdes } \\
\text { es supuesto con } \\
\text { el desarrollo ur- } \\
\text { bano }\end{array}$ & $\begin{array}{l}\text { El desarrollo } \\
\text { de áreas verdes } \\
\text { es supuesto con } \\
\text { el desarrollo ur- } \\
\text { bano }\end{array}$ & $\begin{array}{l}\text { - Escaso descen- } \\
\text { so: entre } 1996 \mathrm{y} \\
2016 \text { la super- } \\
\text { ficie verde pasó } \\
\text { de } 1,86 \mathrm{~km}^{2} \text { a } \\
1,76 \mathrm{~km}^{2} \text {. Aun } \\
\text { así, es baja para } \\
\text { el conjunto ur- } \\
\text { bano } \\
\text { - Sectores sur y } \\
\text { oeste con ma- } \\
\text { yor superficie } \\
\text { verde y presen- } \\
\text { cia de árboles } \\
\text { que sectores } \\
\text { norte y este }\end{array}$ \\
\hline
\end{tabular}

Tabla 1. Desarrollo de los elementos y estructura del sistema ICUPP en cada período histórico de la ciudad de Presidente Prudente Fuente: Elaboración propia. 
La tabla anterior permitió establecer relaciones entre los elementos del sistema ICUPP dentro de un mismo período y en todos los períodos de la historia de la urbe a fin de deducir la evolución del sistema y la influencia que han tenido dichos componentes en el origen y funcionamiento del mismo. En el próximo análisis se consideró, entre dichos componentes, a la trama, la población y las temperaturas de la ciudad, así como el entorno rural inmediato a fin de explicar mejor las diferencias térmicas entre lo urbano y lo rural. Dado que las áreas verdes y la densidad edilicia y demográfica dan cuenta de las diferencias térmicas intraurbanas, las mismas se contemplarán en otro apartado.

Así, en el período 1917-1940 había un escaso desarrollo del espacio urbano, de su población y del área rural cercana, donde aún predominaba la flora primitiva, por lo que, pese a la falta de registros de temperatura de la ciudad, se concluye la existencia poco definida de la isla de calor urbana. En 1941-1959 comenzó cierta expansión de la trama urbana y del número de habitantes, mientras se produjo un importante retroceso del área natural inmediata a favor del desarrollo agrario, lo cual pudo restar frescor al ambiente urbano, llevando a la formación inicial de la isla de calor.

En 1960-1979 el crecimiento urbano y demográfico alcanzó su máxima expresión, al tiempo que continuó el gran avance de la actividad agraria sobre el área de vegetación natural. Todo esto coincidió con el significativo aumento de las temperaturas máximas y mínimas de la ciudad prudentina, lo que lleva a inferir que en este momento se dio la irrupción de la isla de calor como fenómeno claramente definido.

En los últimos períodos, 1980-1999 y 2000-2016, luego de aquel incremento superlativo, la trama y población urbanas han experimentado un reducido aumento y la expansión rural se ha estabilizado, porque no existen amplias superficies naturales por transformar. Pero estos procesos ya no concuerdan con el comportamiento de las temperaturas máximas y mínimas de Presidente Prudente, que han continuado en importante ascenso. Por tanto, en los tiempos recientes la isla de calor prudentina continúa existiendo e incluso va adquiriendo una mayor intensidad.

Ahora bien, la presencia de la malla urbana, la población urbana y el entorno rural próximo determina al sistema ICUPP, pero el escaso crecimiento o estabilidad de 
estos elementos en la actualidad no logran explicar el aumento constante de las temperaturas de la ciudad. Entonces, surgen los interrogantes: ¿a qué se debe dicho aumento de las temperaturas?, ¿qué otro elemento del sistema (que no fue considerado) puede estar actuando? Para encontrar posibles respuestas, en el próximo apartado se retoman los estudios de isla de calor urbana de Presidente Prudente.

\section{Estudios de la isla de calor urbana de presidente prudente como fenómeno mensurable}

Las investigaciones sobre la isla de calor en Presidente Prudente abordan esta realidad como un fenómeno climático posible de constatar a través de la medición y el análisis cuantitativo. En efecto, según Oke (1978, citado por Amorim, 2010), el rasgo más importante de la isla de calor es su intensidad, entendida como la diferencia entre el máximo de temperatura urbana y el mínimo de temperatura rural. Además, es posible distinguir tres tipos de islas de calor: superficial, que se desarrolla en las distintas superficies urbanas; atmosférica inferior, que se halla entre el nivel del suelo y el de los techos edilicios; y atmosférica superior, que ocurre por encima del nivel de los techos.

Para diagnosticar la isla de calor atmosférica inferior, Figura 6, los estudios registraron las temperaturas del aire en la ciudad prudentina y el entorno rural próximo durante el período nocturno ( 21 hs.), cuando no hay cambios térmicos drásticos. Además, eligieron días con baja velocidad de viento, lo cual dificulta la dispersión del calor urbano, y sin precipitaciones, porque estas disminuyen las temperaturas y generan islas de frescor. Estas mediciones se realizaron a lo largo de dos transectos móviles, que recorrieron la urbe en sentido SO-NE y NO-SE, tomando puntos itinerantes de temperatura del aire.

A nivel espacial, el núcleo de la isla de calor urbana corresponde al centro comercial densamente edificado y poco arborizado. Se prolonga hacia el norte y oeste, donde las mayores diferencias de temperatura ocurren en barrios pudientes con alta densidad edilicia y baja cantidad de cubierta vegetal y barrios populares de reducida construcción, pero compacta y ausencia de vegetación. Las temperaturas más bajas suceden en los fondos de valle, áreas con arboledas profusas y barrios cerrados 
situados al sur, que tienen alto patrón constructivo, pero comprenden terrenos amplios y con arbolado.

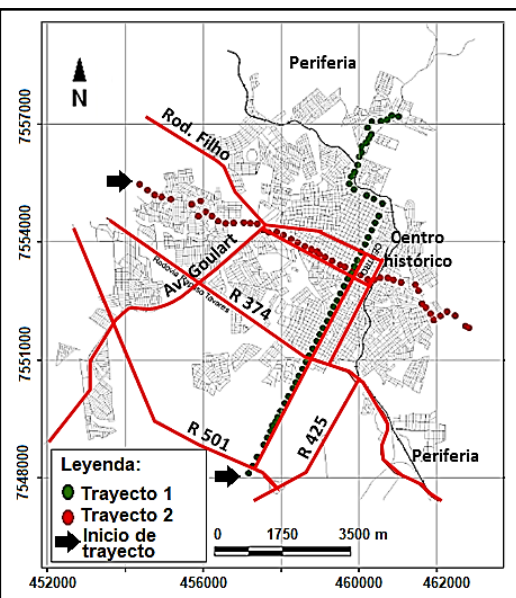

Transectos móviles de medición de temperatura atmosférica

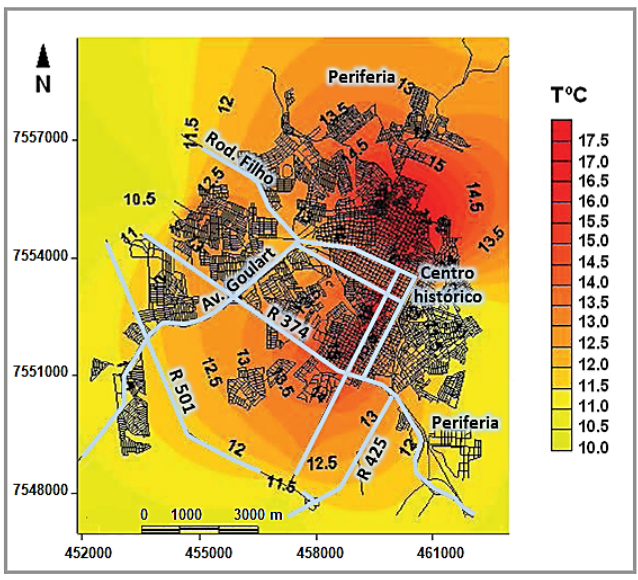

Isla de calor atmosférica inferior según temperaturas registradas el día 30/5/2007 a las $21 \mathrm{hs.}$

Figura 6. Transectos móviles para medir la temperatura atmosférica en Presidente Prudente e isla de calor atmosférica resultante en 2007

Fuente: Elaboración propia, sobre la base de Amorim et al. (2010) y Amorim, M. (2010).

Durante el período 2002-2014 se diagnosticaron islas de calor de fuerte magnitud, las que presentan diferencias de temperatura entre la ciudad y el entorno rural de entre $4^{\circ} \mathrm{C}$ y $6^{\circ} \mathrm{C}$, y muy fuerte magnitud, cuando esas diferencias superan los $6^{\circ} \mathrm{C}$. Pero estos valores de intensidad de la isla de calor prudentina deben contextualizarse según las condiciones sinópticas actuantes. De allí que en la siguiente tabla se diferencie la intensidad de este fenómeno según las dos estaciones del año: amena y seca y cálida y lluviosa. 


\begin{tabular}{|c|c|c|c|c|c|c|}
\hline \multirow{2}{*}{$\begin{array}{l}\text { Estación } \\
\text { del año }\end{array}$} & \multicolumn{6}{|c|}{ Fecha y hora de medición de la intensidad de la isla de calor } \\
\hline & $\begin{array}{c}2002 \\
\text { julio, } 20 \mathrm{hs} .\end{array}$ & $\begin{array}{c}2003 \\
\text { julio, } 20 \text { hs. }\end{array}$ & $\begin{array}{c}2007 \\
\text { mayo, } 21 \text { hs. }\end{array}$ & $\begin{array}{c}2008 \\
\text { octubre, } 15 \text { hs. }\end{array}$ & \begin{tabular}{|c}
2013 \\
diciembre, 21 hs.
\end{tabular} & $\begin{array}{c}2014 \\
\text { junio, } 21 \text { hs. }\end{array}$ \\
\hline $\begin{array}{c}\text { Ameno y } \\
\text { seco } \\
\text { (abril- } \\
\text { septiembre) }\end{array}$ & $6,68^{\circ} \mathrm{C}$ & $8,67^{\circ} \mathrm{C}$ & $7,5^{\circ} \mathrm{C}$ & & & $8,58^{\circ} \mathrm{C}$ \\
\hline $\begin{array}{l}\text { Cálido y } \\
\text { lluvioso } \\
\text { (octubre- } \\
\text { marzo) }\end{array}$ & & & & $2,8^{\circ} \mathrm{C}$ & $4,78^{\circ} \mathrm{C}$ & \\
\hline
\end{tabular}

Tabla 2. Intensidad de la isla de calor de Presidente Prudente desde 2002 hasta 2014

Fuente: Elaboración propia, sobre la base de Amorim, M. (2005), Viana et al. (2007), Amorim et al. (2010), Amorim, M. (2010) y Cardoso, R. (2015).

Por otro lado, la isla de calor superficial se diagnostica mediante imágenes satelitales. Al respecto, los satélites Landsat 5, 7 y 8 , con los sensores del infrarrojo térmico, permiten medir las temperaturas de la superficie, ofreciendo un diseño de temperatura local que posibilita comprender la distribución de las fuentes de calor dentro del área urbana y del rural próximo (Amorim, M., Sant'Anna Neto, J. \& Dubreuil, V., 2008). En Presidente Prudente los estudios sobre isla de calor superficial se realizaron tanto en la estación seca como lluviosa, demostrando la diferente magnitud que aquella adquiere, Figura 7.

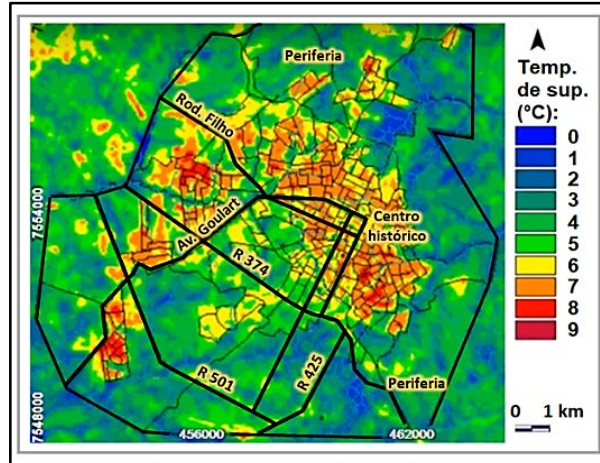

Período lluvioso (30/10/2015)

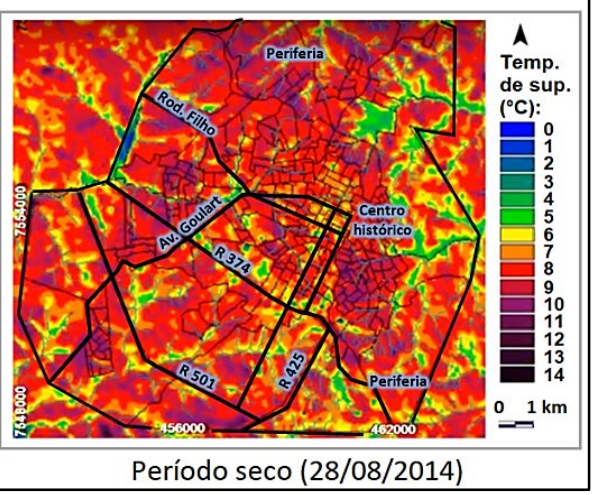

Período seco (28/08/2014)

Figura 7. Isla de calor superficial en Presidente Prudente durante las estaciones seca y lluviosa Fuente: Elaboración propia, sobre la base de Amorim, M. (2017). 
En efecto, durante el período lluvioso la isla de calor superficial es más evidente, debido al marcado contraste entre la menor temperatura rural causada por la presencia de vegetación vigorosa y la mayor temperatura urbana ocasionada por el dominio de coberturas de fibrocemento. Al interior de la ciudad también se distinguen claramente las áreas con densa cubierta arbórea, como los fondos de valle, y las zonas con alto patrón constructivo (Amorim, M., 2017). Mientras, en el período seco la isla de calor superficial disminuye notoriedad, ya que las temperaturas del campo y la ciudad se igualan como producto de la pérdida del follaje y el aumento de suelo expuesto.

\section{Comparación de resultados de la isla de calor como fenómeno complejo y mensurable y surgimiento de nuevos aportes}

A partir de los avances de conocimiento que se lograron con los estudios de la isla de calor de Presidente Prudente tanto desde la visión más desarrollada (como fenómeno mensurable) como desde la propuesta en el presente trabajo (como fenómeno sistémico complejo), se realizó una comparación de los resultados para responder a las preguntas planteadas anteriormente. En esta búsqueda tentativa interesa no tanto el hallazgo de una respuesta acertada, sino más bien el surgimiento de nuevos aportes que puedan ser el punto de partida de investigaciones que profundicen en la isla de calor prudentina

Así, desde el punto de vista espacial, se verifica una fuerte relación entre la isla de calor como fenómeno mensurable y algunos componentes de la isla de calor como sistema complejo, especialmente la densidad edilicia y demográfica y la arborización. De hecho, el área consolidada de Presidente Prudente, correspondiente al centro histórico y sus alrededores, posee un aglomerado de construcciones compactas, varios espacios verdes con importante arboleda (pero reducida superficie) y baja densidad poblacional. Todo ello condice con que este sector sea el núcleo de la isla de calor atmosférica inferior y uno de los sectores de mayor temperatura de la isla de calor superficial.

Por su parte, en la periferia urbana se distinguen dos zonas. La primera está formada por los sectores norte, oeste y este, donde predomina una densidad edilicia media con patrón constructivo compacto, áreas verdes de mediana superficie y con 
vegetación esparcida y densidad demográfica media y alta. Esta zona coincide con otro foco de altas temperaturas de la ciudad, salvo los fondos de valle. La segunda zona comprende el sector sur, que tiene barrios de construcciones más espaciadas, espacios verdes de importante tamaño con arbolado profuso y densidad poblacional media y alta, todo lo cual explica que sea el sector con menor temperatura atmosférica y superficial.

Desde el punto de vista temporal, se compararon los resultados de la isla de calor prudentina como fenómeno complejo y como otro mensurable a través de una tabla, que expone el comportamiento de los principales elementos del sistema ICUPP y de la isla de calor atmosférica inferior a lo largo de la historia de la ciudad.

\begin{tabular}{|c|c|c|c|c|c|c|}
\hline \multirow{2}{*}{\multicolumn{2}{|c|}{$\begin{array}{l}\text { Resultados de dos visiones } \\
\text { de la isla de calor }\end{array}$}} & \multicolumn{5}{|c|}{ Períodos del desarrollo histórico de Presidente Prudente } \\
\hline & & \multirow{2}{*}{$\begin{array}{c}\text { 1917-1940 } \\
-\end{array}$} & \multirow{2}{*}{$\begin{array}{c}\text { 1941-1959 } \\
\uparrow-\end{array}$} & \multirow{2}{*}{$\begin{array}{c}\text { 1960-1979 } \\
\uparrow+\end{array}$} & \multirow{2}{*}{$\frac{\text { 1980-1999 }}{\uparrow-}$} & \multirow{2}{*}{$\frac{\text { 2000-2016 }}{\uparrow-}$} \\
\hline \multirow{4}{*}{$\begin{array}{l}\text { Sistema } \\
\text { ICUPP }\end{array}$} & Trama urbana & & & & & \\
\hline & $\begin{array}{c}\text { Población } \\
\text { urbana }\end{array}$ & - & $\uparrow-$ & $\uparrow+$ & $\uparrow-$ & $\uparrow-$ \\
\hline & Entorno rural & - & $\uparrow+$ & $\uparrow+$ & - & - \\
\hline & $\begin{array}{c}\text { Temperaturas } \\
\text { máximas y } \\
\text { mínimas de } \\
\text { ciudad }\end{array}$ & & & $\begin{array}{l}\uparrow+\left(t^{\circ} \text { min }\right) \\
\uparrow+\left(t^{\circ} \text { máx }\right)\end{array}$ & $\begin{array}{l}\uparrow+\left(t^{\circ} \text { min }\right) \\
\uparrow+\left(t^{\circ} \text { máx }\right)\end{array}$ & $\begin{array}{r}\uparrow+\left(t^{\circ} \text { min }\right) \\
\uparrow+\left(t^{\circ} \text { máx }\right)\end{array}$ \\
\hline $\begin{array}{l}\text { Isla de } \\
\text { calor } \\
\text { atmosférica } \\
\text { inferior }\end{array}$ & $\begin{array}{c}\text { Diferencia } \\
\text { entre la } \\
\text { máxima } t^{\circ} \\
\text { urbana y la } \\
\text { mínima } t^{\circ} \text { rural }\end{array}$ & & & & & $\begin{array}{c}\uparrow \downarrow \uparrow \\
\text { (comporta- } \\
\text { miento } \\
\text { variable } \\
\text { según las } \\
\text { condiciones } \\
\text { del tiempo) }\end{array}$ \\
\hline \multicolumn{2}{|c|}{ REFERENCIAS } & $\begin{array}{c}\uparrow+ \\
\text { aumento } \\
\text { creciente }\end{array}$ & $\begin{array}{c}\uparrow- \\
\text { aumento } \\
\text { decreciente }\end{array}$ & $\stackrel{\downarrow}{\text { disminución }}$ & $\underset{\text { estabilidad }}{-}$ & \\
\hline
\end{tabular}

Tabla 3. Evolución de los principales elementos del sistema ICUPP y de la isla de calor atmosférica inferior a través de la historia de Presidente Prudente

Fuente: Elaboración propia. 
Al observar la tabla y en respuesta a las preguntas formuladas, se concluye que en las últimas décadas el aumento constante de las temperaturas máximas y mínimas de la urbe no puede explicarse por la trama urbana, la población urbana y el entorno rural próximo, como tampoco por la diferencia térmica entre campo y ciudad, ya que el comportamiento temporal de estos aspectos no coincide con ese ascenso térmico.

Otro factor que puede explicar este fenómeno es la movilidad espacial de la población, ya que implica el empleo de vehículos como fuente generadora de calor. Aunque no se tengan registros estadísticos de esta variable, es probable que su influencia en el aumento de temperaturas de Presidente Prudente sea relativa, porque el tamaño poblacional medio de la ciudad supone un uso del transporte muy inferior al que se realiza en las grandes urbes. De allí, que en este punto del hallazgo, cabe plantearse la posible actuación de cambios ocurridos en el nivel superior de la atmósfera, lo cual es difícil de evaluar por las costosas técnicas de medición que implica su estudio.

Pese a ello, el siguiente raciocinio permitirá hacer un proceso deductivo para aceptar o rechazar aquel supuesto: si se considera que la isla de calor surge de la diferencia entre la temperatura más alta ocurrida en la ciudad y la más baja sucedida en el medio rural cercano, la evolución de esa intensidad en un período de tiempo prolongado puede ser reveladora. Para ello es importante llevar un registro continuo de datos de temperatura tanto en el medio urbano como en el rural, pero no solo del entorno próximo, sino también del alejado de la ciudad, que represente, por un lado, al ambiente rural (dominio de cultivos), y por otro, al natural primitivo (ej. Reserva Natural Mata do Furquim).

En estas condiciones es posible lograr un análisis histórico que arroje dos resultados distintos con su correspondiente deducción. Si la diferencia de temperatura muestra un aumento significativo en el período analizado, entonces es el fenómeno urbano (con sus construcciones, falta de arborización, etc.) que difiere claramente del rural y es un factor decisivo en el aumento de las temperaturas de Presidente Prudente, llevando a la formación de un verdadero clima urbano. Pero si esa intensidad energética tiene poco aumento o permanece igual, entonces ya no es lo urbano distintivo de lo rural y, por ende, un factor extra local está actuando en el ascenso de la temperatura urbana. 


\section{CONCLUSIONES}

El abordaje sistémico complejo de la isla de calor urbana en la ciudad de Presidente Prudente demuestra una contribución metodológica innovadora que complementa los estudios analíticos realizados hasta el momento, porque permite observar el conjunto de la problemática y, al mismo tiempo, sus componentes a lo largo de los períodos históricos de la urbe. Esa diversidad y totalidad de una misma realidad, junto con los resultados comparativos, permitieron observar coincidencias a nivel espacial de algunos aspectos constitutivos de la isla de calor tanto como fenómeno mensurable como complejo y encontrar diferencias a nivel temporal de estas dos visiones en el intento de explicar el aumento constante de las temperaturas de la ciudad. Este último punto inconcluso llevó a crear interrogantes y supuestos a fin no tanto de hallar respuestas acertadas, sino más bien abrir nuevos caminos para profundizar en este complejo problema urbano-ambiental.

\section{BIBLIOGRAFÍA}

Amorim, M. (2000). O clima urbano de Presidente Prudente S/P. Tesis de Doctorado para optar por el título de Doctora en Geografía, San Pablo: Facultad de Filosofía, Letras e Ciencias Humanas, Universidad de San Pablo.

Amorim, M., Sant'Anna Neto, J. \& Dubreuil, V. (2008, agosto). A utilização do canal termal do Landsat 7 e de transectos móveis para o estudo de clima urbano. VIII Simposio Brasileiro de Climatología Geográfica, Alto Caparaó, Brasil.

Amorim, M. (2010), Climatologia e gestão do espaço urbano. Mercator, dezembro, 71-90.

Amorim, M., Teodoro, P., Braido, L., Montezani, E., Rivero, C., Souza, C., Menezes, B., Aleixo, N. \& Araujo, A. (2010), Ensaio metodológico sobre a utilização de transectos móveis no período diurno em Presidente Prudente - SP. Formação, (1), 77-95.

Amorim, M. (2017). Teoria e método para o estudo das ilhas de calor em cidades tropicais de pequeno e médio porte. Tesis presentada para obtener el título de Libre-Docente en "Climatología Geográfica", Facultad de Ciencias y Tecnología, Universidad Estadual Paulista, Presidente Prudente.

Caccia Gouveia, J. \& Paes, J. (2017). Cobertura vegetal. Atlas Ambiental Escolar de Presidente Prudente-SP, Brasil. Recuperado de http://portaldoprofessor.fct.unesp.br:9000/topico/meiofisicobiotico/ 
Cardoso, R. (2015). Classificação de potencias unidades climáticas em Presidente Prudente $S P$. Disertación para optar por el título de Magíster en Geografía, Presidente Prudente: Facultad de Ciencias y Tecnología, Universidad Estadual Paulista.

Codes, I. \& Robledo, S. (1997). Propuesta para la enseñanza de la problemática medioambiental en la EGB y Polimodal. En Capitanelli, R. (Ed), Problemas del medio ambiente de la provincia de Mendoza (pp. 177-190). Mendoza: Ecogeo.

Domizio, M. C. (2018). Una problemática puntual abordada desde un enfoque integral: la relación sociedad-arbolado de calle de la ciudad de Mendoza bajo una mirada sistémica compleja. VI Encuentro Latinoamericano de Metodología de las Ciencias Sociales, Cuenca, Ecuador.

Fante, K. (2014). Variabilidade da temperatura em áreas urbanas não metropolitanas do Estado de São Paulo-Brasil no período de 1961 a 2011. Disertación para optar por el título de Magíster en Geografía, Presidente Prudente: Facultad de Ciencias y Tecnología, Universidad Estadual Paulista.

García, R. (2006). Sistemas complejos. Conceptos, métodos y fundamentación epistemológica de la investigación interdisciplinaria. Barcelona: Gedisa.

Gomes, M. \& Amorim, M. (2002). As praças públicas de Presidente Prudente/SP: dinámica sócio-espacial e caracterização da vegetação. Geografía em atos, 21-37.

Melazo, E., Cardoso, J. \& Magaldi, S. (2017). Indicadores socioespaciais. Atlas Ambiental Escolar de Presidente Prudente-SP, Brasil. Recuperado de http://portaldoprofessor.fct.unesp .br:9000/topico/infra-estrutura/

Menotti, S., Camargo, C., Amorim, M. \& Sant'Anna Neto, J. (2004). Estudo de ilhas de calor em Presidente Prudente/SP a partir de transecto móveis. Formação, (2), 35-62.

Nunes, J.; Freire, R. \& Peres, I. (2006, setembro). Mapeamento geomorfológico do perímetro urbano de Presidente Prudente-SP, VI Simposio Nacional de Geomorfología, Goiana, Brasil.

Recuperado de http://www.labogef.iesa.ufg.br/links/sinageo/aut/articles/440.pdf

Pedro, Leda (2014). Dinâmicas de apropiação e ocupação em diferentes formas de relevo: Análise dos impactos e vulnerabilidade nas cidades de Presidente Prudente/SP e Marília/SP. Tesis de Doctorado para optar por el título de Doctora en Geografía, San Pablo: Facultad de Filosofía, Letras y Ciencias Humanas, Universidad de San Pablo.

Pereira, S. (2006), Expansão e estructuração interna do espaço urbano de Presidente Prudente-SP, Formação, (2), 55-72. 
Souza, M. (2016). Diagnóstico da qualidade ambiental nas áreas verdes públicas em Presidente Prudente (SP). Disertación para optar por el título de Magíster en Geografía, Presidente Prudente: Facultad de Ciencias y Tecnología, Universidad Estadual Paulista.

Veloso, H., Rangel Filho, A. \& Lima, J. (1991). Classificação da vegetação brasileira, adaptada a um sistema universal. Río de Janeiro: Departamento de Recursos Naturales y Estudios Ambientales, Instituto Brasilero de Geografía y Estadística.

Viana, S., Camargo, C., Amorim, M. \& Sant'Anna Neto, J. (2007). Estudo de ilhas de calor em Presidente Prudente/SP a partir de transectos móveis. Formação, (13/12), 431-458.

Whitacker, A. (2017). Espaço Urbano, Atlas Ambiental Escolar de Presidente Prudente-SP, Brasil. Recuperado de http://portaldoprofessor.fct.unesp.br:9000/topico/formacao-socio espacial/

\section{Otras fuentes consultadas}

Instituto Brasilero de Geografía Estadística (IBGE): https://sidra.ibge.gov.br/

Estación Meteorológica de Presidente Prudente, Universidad Estadual Paulista

Secretaría Municipal de Planeamiento, Desarrollo Urbano y Habitación, Prefectura de Presidente Prudente

\section{LA AUTORA}

María Cecilia Domizio es Técnica en Cartografía, SIG y Teledetección y Licenciada en Geografía por la Universidad Nacional de Cuyo y desarrolla su tesis doctoral en la misma casa de estudios. Ha sido miembro de proyectos de investigación de esta institución y del Instituto de Ciencias Humanas, Sociales y Ambientales (INCIHUSA), CONICET. Actualmente es Profesora de Trabajos Prácticos de la cátedra Metodología de la Investigación en Geografía, Facultad de Filosofía y Letras, Universidad Nacional de Cuyo. Su actividad científica se ha desarrollado en torno a problemáticas urbano-ambientales, en general, y al arbolado público y su gestión municipal en la ciudad de Mendoza, en lo específico. Sus publicaciones en revistas y congresos nacionales e internacionales se han inscripto en la línea de aplicación de la Teoría de los Sistemas Complejos como enfoque teórico-metodológico para abordar la compleja realidad territorial, vinculando fenómenos y procesos de origen social y natural, y para entablar diálogos complementarios con el municipio. Ha obtenido una beca de estancia investigativa en la Universidad Estadual Paulista, situada en la ciudad de Presidente Prudente, Brasil, cuya labor da cuenta el presente trabajo.

ceciliadomizio@ffyl.uncu.edu.ar 